\title{
Análisis de tendencia de temperatura y precipitación para el departamento de Caldas (Colombia), mediante wavelets*
}

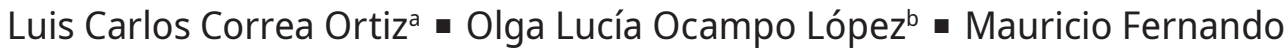 \\ Alba Castroc
}

\begin{abstract}
Resumen: el cambio climático, entendido como una alteración del clima, debida o no a la actividad humana, sumada a la variabilidad climática, es un área de estudio primordial en estos momentos. Este estudio se hizo con el objetivo de generar información de tendencias de las variables temperatura y precipitación para el departamento de Caldas, Colombia, mediante la técnica de análisis wavelets. Para tal fin, se seleccionaron las estaciones del Ideam, ubicadas en el territorio, para los periodos 1971-2010, en el caso de precipitación; y para el periodo 1981-2010, en temperatura. Las estaciones registran datos diariamente, los cuales fueron transformados en datos mensuales, cuya tendencia se calculó mediante el análisis multirresolución (MRA), seleccionando la wavelet Daubechies (dB5) con seis niveles de descomposición. Los análisis evidencian tendencias lentas y crecientes en precipitación para las quince estaciones analizadas. Resultado similar se obtuvo para temperatura mínima. Mientras que, para temperatura máxima, se registraron tendencias lentas decrecientes, en una estación. Para los planes integrales de gestión de cambio climático, se recomienda el seguimiento a estas tendencias, en lo posible con series históricas de mayor longitud, para identificar señales de cambio y posibles efectos en el clima regional.
\end{abstract}

Palabras clave: análisis de tendencia; cambio climático; precipitación; temperatura; wavelets.

Recibido: 10/06/2020 Aceptado: 20/01/2021 Disponible en línea: 23/07/2021

* Artículo de investigación, que presenta parte de los resultados del trabajo de grado "Plataforma sIG de Cambio Climático para el departamento de Caldas", realizado como requisito parcial para optar al título de Magíster en Ingeniería de la Universidad Autónoma de Manizales.

a Ingeniero electrónico, magíster en Educación y Desarrollo Humano, magíster en Ingeniería. Profesor asociado Facultad de Ciencias e Ingeniería, Universidad de Manizales, Manizales, Colombia. Correo electrónico: Icco@umanizales.edu.co ORCID: https://orcid.org/0000-0001-9488-5249

b Ingeniera química, magíster en Ingeniería Química, doctora en Ingeniería. Docente asociada del Departamento de Mecánica y Producción, Universidad Autónoma de Manizales, Manizales, Colombia. Correo electrónico: olocampo@autonoma.edu.co ORCID: https://orcid.org/0000-0002-6394-977X

c Ingeniero de sistemas y computación, doctor en Programación Declarativa e Ingeniería de la Programación. Docente titular del Departamento de Ciencias Computacionales, Universidad Autónoma de Manizales, Manizales, Colombia. Correo electrónico: malba@autonoma.edu.co ORCID: https://orcid. org/0000-0001-5419-1621 
Cómo citar: L. C. Correa Ortiz, O. L. Ocampo López, y M. F. Alba Castro, «Análisis de tendencia de temperatura y precipitación para el departamento de Caldas (Colombia), mediante wavelets», Cien. Ing.Neogranadina, vol. 31, n. 1, pp. 37-52, jul. 2021.

\title{
Temperature and precipitation trend analysis for the department of Caldas (Colombia), using wavelets
}

\begin{abstract}
Climate change, understood as an alteration of the climate, due or not to human activity, added to climate variability, is a primary area of study at this time. This study was carried out with the objective of generating information on trends of the temperature and precipitation variables for the department of Caldas, Colombia, using the wavelets analysis technique. For this purpose, the Ideam stations, located in the territory, were selected for the periods 1971-2010, in the case of precipitation; and for the period 1981-2010, for temperature. The stations record data daily, which was transformed into monthly data, their trend was calculated by multi resolution analysis (MRA), selecting the Daubechies wavelet (dB5) with six levels of degradation. The analysis shows slow and increasing trends in precipitation for the fifteen stations analyzed. A similar result was obtained for minimum temperature. While, for maximum temperature, slow decreasing trends were recorded in one station. For comprehensive climate change management plans, it is recommended to monitor these trends, if possible with longer historical series, to identify signs of change and possible effects on the regional climate.
\end{abstract}

Keywords: trend analysis; climate change; precipitation; temperature; wavelets. 


\section{Introducción}

Al hablar de Colombia, emergen varios temas: biodiversidad, dos mares, variedad de pisos térmicos, todos los climas; un país emergente; con cierta bonanza petrolera y minera; recursos naturales en abundancia e importante inversión extranjera, entre otros. Sin embargo, el país no logra un desarrollo sostenible que permita disminuir la desigualdad - puesto 79 en el mundo [1] - y sacar a millones de sus habitantes de la pobreza, sin detrimento de los recursos naturales; particularmente, por la ausencia de políticas de largo plazo que faciliten al país una adecuada planificación.

Si bien, se han dado pasos importantes en esta dirección con los compromisos internacionales relacionados con desarrollo sostenible, como la Agenda 2030 y sus Objetivos de Desarrollo Sostenible (oDs), la implementación del Acuerdo de París sobre Cambio Climático y las recomendaciones de la Organización para la Cooperación y el Desarrollo Económicos (OCDE), como lo establece la Política de Crecimiento Verde [2]; los retos cada día son mayores por la vulnerabilidad del territorio a la variabilidad y el cambio climáticos.

El cambio climático es una realidad. Cada vez más los fenómenos asociados a la variabilidad climática tienen mayor intensidad: basta con dar un vistazo a las noticias para evidenciar drásticos aumentos y disminuciones de temperatura, sin contar con variaciones en la precipitación, que traen consigo múltiples desastres naturales (inundaciones y sequías), así como impacto en la agricultura [3]. Colombia no es la excepción y, si bien la política pública de los ámbitos internacional y nacional, ligada al manejo del riesgo que involucran estos fenómenos, se ha fortalecido, es mucho lo que resta por hacer en cuanto a adaptación y mitigación de los efectos del cambio climático en el país, por su grado de vulnerabilidad.

Recientemente, debido al fenómeno climático de El Niño, el país se vio sometido a una crisis sin precedentes, con grandes pérdidas en el sector agropecuario, debido a la sequía generada por este fenómeno [4]. También estuvo ad portas de un racionamiento eléctrico e hídrico, por el bajo nivel de ríos y embalses usados para la alimentación de los acueductos de los municipios y la generación hidroeléctrica. Particularmente en el departamento de Caldas, el 75,22\% de los cultivos de café - uno de los productos tradicionales - se vio afectado por el evento de El Niño, mientras las cifras de pérdidas en ganadería tampoco fueron alentadoras (300000 millones de pesos colombianos -COP, aproximadamente [5]).

A pesar de que este fenómeno es cíclico, se evidencia la falta de información que permita tomar decisiones efectivas, con base en el comportamiento, en general, del clima y la temperatura y la precipitación, en particular, en años futuros. Contar con esta información permitirá generar análisis, planes, programas y políticas públicas para la mitigación y posterior adaptación ante el cambio climático, como en el caso de los caficultores de la cuenca del río Chinchiná, en Caldas [6], [7]; políticas en las que se incluya el análisis más allá de lo físico-climático, considerando las dimensiones sociales, políticas y culturales del fenómeno [8].

Aquí, es importante destacar la labor del Instituto de Hidrología, Meteorología y Estudios Ambientales (Ideam), el cual en 2015 publicó su Tercera comunicación nacional de cambio climático, donde se incluyeron nuevos escenarios de cambio climático para Colombia, a nivel departamental [9].

A partir de lo expuesto, el presente estudio se enfoca en realizar un análisis de tendencia de las variables temperatura y precipitación en los periodos 1971-2010 y 1981-2010, respectivamente.

\section{Temperatura, precipitación y cambio climático}

La temperatura es uno de los parámetros que hacen parte de la medición del clima [10]. En este sentido, el cambio climático ejerce influencia en los cambios en la temperatura. Se señala que esta última es "el resultado de un complejo equilibrio de energía, debido a las radiaciones solares, a la composición de la atmósfera, a los cambios en los continentes, a las corrientes oceánicas y a la órbita de la tierra" [11, p. 73]. Por tanto, la pérdida de este equilibrio origina cambios climáticos.

Se considera que el cambio de la temperatura es uno de los efectos esenciales del cambio climático 
[10]. Al respecto, investigadores como L. Alexander et al. [12] analizaron datos de la temperatura diaria en diferentes países en 1951-2003. Encontraron que, en el $70 \%$ de las áreas analizadas de la tierra, durante este periodo, se produjo un calentamiento (incremento de las temperaturas extremas asociadas, especialmente, con calor). De igual forma, en la investigación de análisis de escenarios, adscrita a los reportes del Intergovernmental Panel on Climate Change (IPCC), conjeturan que los cambios de temperatura no serán uniformes en las regiones del mundo [13]. Sin embargo, con altos niveles de confianza, aseguran que la región ártica experimentará un incremento importante de temperaturas.

Asimismo, el análisis de escenarios realizado muestra que las temperaturas medias de la atmósfera sufrirán calentamiento en toda la tropósfera, especialmente en la tropósfera alta y en las latitudes más septentrionales [13]. Contrariamente, se espera una disminución de la temperatura en la estratósfera. Las investigaciones realizadas por el Ideam, para Colombia, calculó un incremento de la temperatura media de un orden de $0,13{ }^{\circ} \mathrm{C}$ por década en el periodo 1971-2000, mientras los escenarios proyectan que la temperatura promedio del aire en el país aumentará, con respecto al período de referencia $1971-2000$, en $1,4^{\circ} \mathrm{C}$ para el 2011-2040; en $2,4^{\circ} \mathrm{C}$, para $2041-2070$; y $3,2^{\circ} \mathrm{C}$ para el 2071-2100 [14].

Las precipitaciones también hacen parte de las dimensiones del clima [11]. En el contexto del cambio climático, de acuerdo con simulaciones del IPCC [10], los eventos de precipitaciones intensas se incrementarán, si se mantiene el aumento de los gases de efecto invernadero. Efectivamente, se ha construido evidencia de que el cambio climático, especialmente el influido por el ser humano, está transformando las precipitaciones y el ciclo hidrológico [13]. Esto ha generado que, en algunos espacios terrestres, las precipitaciones sean extremas y se produzcan inundaciones, mientras que, en territorios donde solía llover, el fenómeno de la lluvia se ha reducido [15]. Por tanto, el cambio en las precipitaciones ha generado cambios de patrones con respecto a los fenómenos de sequía e inundación en muchas regiones [7].
Existe dependencia de la sensibilidad de las precipitaciones al cambio de temperatura por cada grado [16]. En este sentido, la relación entre las precipitaciones y la temperatura global es aproximadamente lineal [13]. El cambio de precipitaciones puede estar en 1-3\% por cada grado de variación en la temperatura (relación establecida para el siglo Xxi). En Colombia, Corredor [17] presenta estudios sobre precipitación máxima en Colombia; y Corredor y Peñaranda [18] dan a conocer un análisis espacial de precipitación en la cuenca alta del río Bogotá. A su turno, el Ideam estimó que, para el presente siglo, los volúmenes de precipitación decrecerían en 15-36\% para amplias zonas de las regiones Caribe y Andina; mientras que existirían incrementos de precipitación hacia el centro y norte de la Región Pacífica [14].

\section{Análisis de tendencia en variables climatológicas y wavelets}

En términos generales, el análisis de tendencia alude a la recolección y posterior procesamiento de la información asociada a un fenómeno, con el fin de evidenciar un patrón, dinámica o comportamiento. Si bien se encuentra relacionado con la predicción del comportamiento del fenómeno analizado, es útil también para describir e identificar dicho comportamiento en el pasado y el presente [19].

Existe una amplia gama de modelos estadísticos para realizar pronósticos que varían en su nivel de complejidad, desde los modelos propuestos por la estadística clásica, pasando por la regresión, hasta abarcar los modelos de series temporales que involucran, incluso, redes neuronales. Todos ellos, sin embargo, requieren una apreciable cantidad de información confiable, caracterizada y normalizada adecuadamente, para producir una buena estimación [20].

Para el caso concreto de series climatológicas, las características previamente descritas, en relación con la información, no se cumplen a cabalidad; pues se presentan deficiencias tanto en calidad como en cantidad: datos faltantes, cambios y datos atípicos [21]. Esto demanda un análisis 
exploratorio - gráfico y cuantitativo- de los datos, antes de realizar cualquier tipo de análisis. Es importante mencionar, además, que dichos análisis no solo son de naturaleza temporal sino espacial. Diferentes estudios realizan estos análisis de tendencias; es así como Velasco y Granados [22] realizaron un análisis de tendencia en precipitación para la cuenca alta y media del río Magdalena.

El análisis de tendencia es particularmente difícil cuando se tiene una serie de tiempo de naturaleza no estacionaria, como en este caso. Por ello, resulta más útil evaluar tanto la tendencia como los ciclos estacionarios de la serie de forma separada. De allí, que métodos tradicionalmente usados en el análisis de señales tengan aplicabilidad en estos casos, tales como la transformada de Fourier en tiempo discreto (DTFT), la transformada de Fourier a corto plazo (STFT) y la transformada wavelet (WT) [23].

En particular, la wT permite realizar un análisis en tiempo frente a frecuencia de la serie de tiempo. La teoría que subyace a la WT es similar a la STFT, con la diferencia fundamental que ofrece una función ventana flexible, denominada wavelet madre, la cual puede adaptarse a las características de la señal; mientras que la STFT representa un método ineficiente para el análisis tiempo frente a frecuencia, al tener una ventana fija, lo que genera solapamiento de los componentes de baja y alta frecuencia que no estén en el rango de frecuencia de la ventana [24].

Debido a que la información climatológica, precipitación y temperatura para este caso es de naturaleza discreta, se usa la transformada wavelet discreta (DWT). La DWT divide la señal en dos: por una parte, la tendencia, copia de la señal original, pero con menor resolución (información de baja frecuencia, llamada también aproximaciones, A). Por otra, las fluctuaciones, que guardan información sobre la diferencia entre la señal modificada y la señal original (altas frecuencias, variaciones en intervalos cortos de tiempo, también denominadas detalles, $D$ ).

Los dos tipos de wavelet madre más utilizados en estudios meteorológicos son las wavelets Daubechies y Morlet [25]. A continuación, se presenta un breve desarrollo matemático alrededor de la DWT, con especial énfasis en las wavelets Haar y Daubechies, las usadas en el presente trabajo y descritas en Oliveira [26].

Sea $f$ una serie discreta, o lo que es lo mismo, $f=\left\{f_{1}, f_{2}, \ldots, f_{N}\right\} \in R^{N}$ con $N=2^{k}$, donde k es lo suficientemente grande. La serie promedio de Haar de primer nivel puede definirse como una nueva señal $A^{1}$, que se obtiene al promediar los elementos de $f$ dos a dos y sustituyendo los valores que se promedian por sus respectivos promedios. La serie en detalle de Haar de primer nivel puede definirse como $D^{1}=f-A^{1}$, la diferencia entre la señal original y la calculada promedio calculado. Por ejemplo, si $f=\{2,5,7,3\}, A^{1}=\{3,5 ; 3,5 ; 5 ; 5\}$. La nueva serie tiene una menor resolución que la serie original. $D^{1}=\{-1,5 ; 1,5 ; 2 ;-2\}$.

La serie Haar de segundo nivel podrá calcularse promediando los cuatro elementos ya sea de $f$ o de $A^{1}, A^{2}=\{4,25 ; 425 ; 4,25 ; 4,25\}$ y la serie en detalle de Haar de segundo nivel será $D^{2}=A^{1}-A^{2}=\{-0,75 ;-0,75 ; 0,75 ; 0,75\}$.

Finalmente, la serie original podrá reconstruirse como $f=A^{2}+D^{2}+D^{1}[21]$. Lo descrito mediante este sencillo ejemplo es el eje central de los análisis con wavelets y se conoce como análisis de multirresolución (MRA, por sus siglas en inglés).

En mayor detalle, sea $\left\{v_{1}^{1}, \ldots, v_{N / 2}^{1}, w_{1}^{1}, \ldots, w_{N / 2}^{1}\right\}$ una base ortonormal en $R^{N} \mathrm{y}$

$$
\begin{gathered}
f=\left(f \cdot v_{1}^{1}\right) v_{1}^{1}+\cdots+\left(f \cdot v_{N / 2}^{1}\right) v_{N / 2}^{1}+\left(f \cdot w_{1}^{1}\right) w_{1}^{1}+\cdots+\left(f \cdot w_{N / 2}^{1}\right) w_{N / 2}^{1} \\
f=a_{1} v_{1}^{1}+\cdots+a_{N / 2} v_{N / 2}^{1}+d_{1} w_{1}^{1}+\cdots+d_{N / 2} w_{N / 2}^{1}
\end{gathered}
$$

Esto es $f=A^{1}+D^{1}$, el primer nivel de MrA de Haar, donde $A^{1}$ es la primera serie promedio (ecuación 1).

$$
A^{1}=\sum_{j=1}^{N / 2}\left(f \cdot v_{j}^{1}\right) v_{j}^{1}
$$

Además, $D^{1}$ es la serie del primer detalle (ecuación 2).

$$
D^{1}=\sum_{j=1}^{N / 2}\left(f \cdot w_{j}^{1}\right) w_{j}^{1}
$$

En el segundo nivel del Mra de $f$, $f=A^{2}+D^{2}+D^{1}$, donde $A^{2}$ puede expresarse mediante la ecuación 3 .

$$
A^{2}=\sum_{j=1}^{N / 4}\left(f \cdot v_{j}^{2}\right) v_{j}^{2}
$$


La serie del segundo detalle en la ecuación 4.

$$
D^{2}=\sum_{j=1}^{N / 4}\left(f \cdot w_{j}^{2}\right) w_{j}^{2}
$$

Esto puede continuar de forma inductiva hasta llegar a un MrA de nivel $m$ tal que $f=A^{m}+$ $D^{m}+\cdots+D^{2}+D^{1}$, donde $m \leq k$ y $N=2^{k}$. Si se desea recuperar la señal original, bastará tomar la

$$
\begin{gathered}
v_{1}^{1}:=\left\{\alpha_{1}, \alpha_{2}, \alpha_{3}, \alpha_{4}, 0, \ldots, 0\right\} \\
v_{2}^{1}:=\left\{0,0, \alpha_{1}, \alpha_{2}, \alpha_{3}, \alpha_{4}, 0, \ldots, 0\right\} \\
\vdots \\
v_{N / 2-1}^{1}:=\left\{0, \ldots, 0, \alpha_{1}, \alpha_{2}, \alpha_{3}, \alpha_{4}\right\} \\
v_{N / 2}^{1}:=\left\{\alpha_{3}, \alpha_{4}, 0, \ldots, 0, \alpha_{1}, \alpha_{2}\right\}
\end{gathered}
$$

Los coeficientes de escalamiento son:

$$
\alpha_{1}=\frac{1+\sqrt{3}}{4 \sqrt{2}} \quad \alpha_{2}=\frac{3+\sqrt{3}}{4 \sqrt{2}}
$$

Y los coeficientes wavelets son:

$$
\beta_{1}=\alpha_{4} \quad \beta_{2}=-\alpha_{3}
$$

Las subseries de tendencia $A^{1}$ y las de fluctuaciones $D^{1}$ se definen igual que en Haar $\operatorname{los} a_{j}^{1}$ son una media ponderada de los cuatro elementos consecutivos de la serie $f$ mientras que los $d_{j}^{1}$ son una diferencia ponderada para $i=1,2, \ldots, N / 2$. Finalmente, de forma inductiva pueden deducirse las wavelets Daubechies de niveles superiores (dbJ) con $J=4,5, \ldots, 10$ [26]. serie promedio más baja, $A^{k}$, y agregarle los detalles $D^{k}, D^{k-1}, \ldots, D^{1}$. En la descomposición MRA para cada $f$ solo se calculan las tendencias $a_{j}$, y las fluctuaciones $d_{j}$, para $1 \leq j \leq 2^{\frac{N}{2 m}}$ [26].

El concepto previo se aplica para las wavelets de Daubechies, comenzando en el nivel 2 (db2), en el cual se define una base ortonormal $\left\{v_{1}^{1}, \ldots, v_{N / 2}^{1}, w_{1}^{1}, \ldots, w_{N / 2}^{1}\right\}$, donde:

$$
\begin{aligned}
w_{1}^{1}:=\left\{\beta_{1}, \beta_{2}, \beta_{3}, \beta_{4}, 0, \ldots, 0\right\} \\
w_{1}^{1}:=\left\{\beta_{1}, \beta_{2}, \beta_{3}, \beta_{4}, 0, \ldots, 0\right\} \\
\vdots \\
w_{N / 2-1}^{1}:=\left\{0, \ldots, 0, \beta_{1}, \beta_{2}, \beta_{3}, \beta_{4}\right\} \\
w_{N / 2}^{1}:=\left\{\beta_{3}, \beta_{4}, 0, \ldots, 0, \beta_{1}, \beta_{2}\right\}
\end{aligned}
$$

$$
\alpha_{3}=\frac{3-\sqrt{3}}{4 \sqrt{2}} \quad \alpha_{4}=\frac{1-\sqrt{3}}{4 \sqrt{2}}
$$

$$
\beta_{3}=\alpha_{2} \quad \beta_{4}=-\alpha_{1}
$$

\section{Metodología}

Inicialmente, fue necesario seleccionar las estaciones del Ideam para el departamento de Caldas. El criterio de selección de las estaciones fue que tuviesen información recolectada en el periodo 19712010, para el caso de precipitación, y en el periodo 1981-2010, para la temperatura. La tabla 1 consigna las estaciones consideradas en el presente estudio; mientras que la figura 1 muestra su ubicación. 


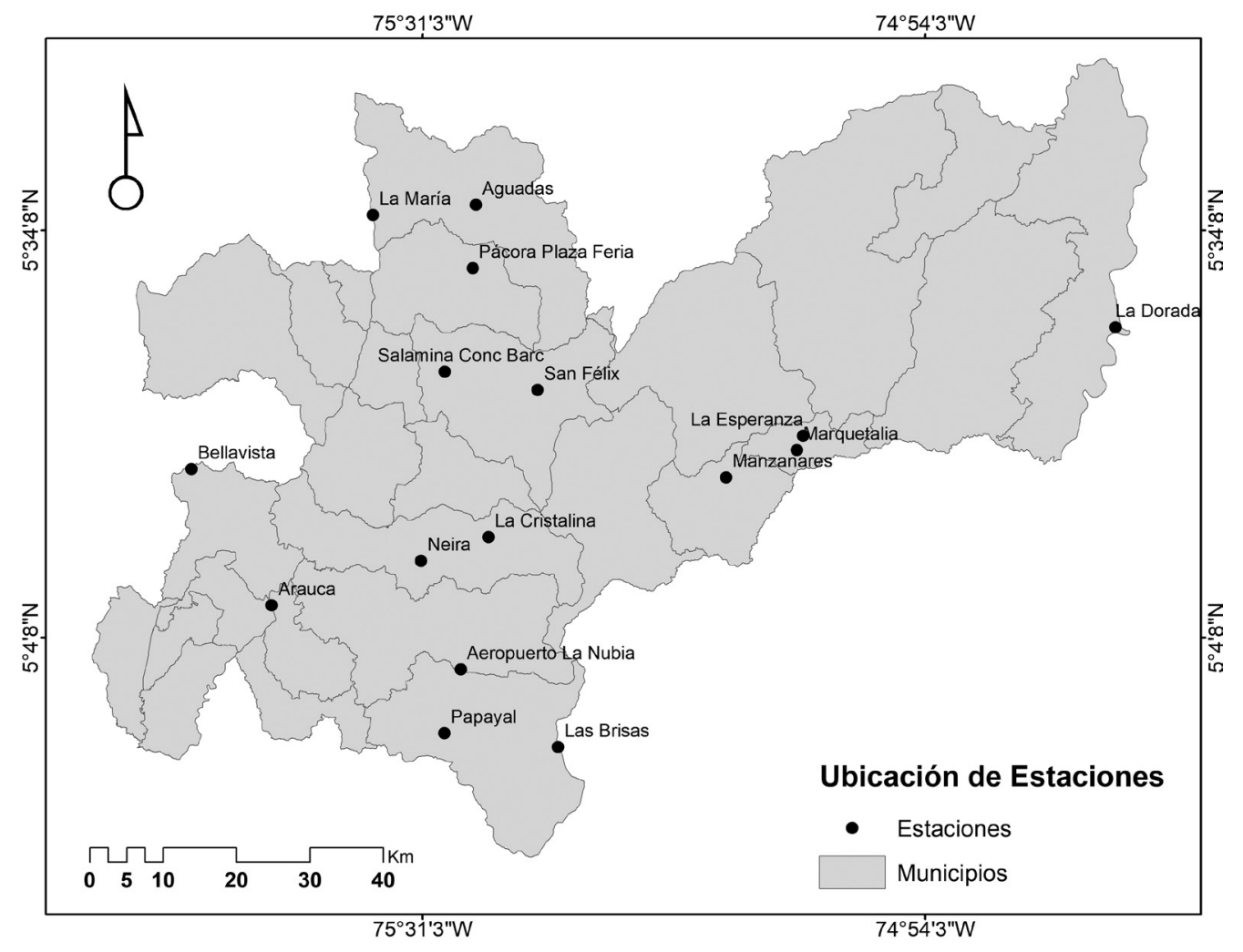

Figura 1. Mapa de localización de las estaciones.

Fuente: elaboración propia.

Tabla 1. Listado de estaciones incluidas en este estudio

a. Precipitación

\begin{tabular}{llllllll} 
Código & Nombre & Tipo & Municipio & Coordenadas & Altitud & $\begin{array}{l}\text { Fecha de } \\
\text { instalación }\end{array}$ \\
\hline 26155110 & Aeropuerto La Nubia & Sinóptica Principal & Manizales & $(5,02977778,75,46991667)$ & 2104 & $15-06-68$ \\
\hline 26180190 & Aguadas & Pluviométrica & Aguadas & $(5,60002778,75,45119444)$ & 2400 & $15-08-70$ \\
\hline 26150060 & Arauca & Pluviométrica & Palestina & $(5,10852778,75,70166667)$ & 917 & $15-02-62$ \\
\hline 26145020 & Bellavista & Climática principal & Anserma & $(5,27594444,75,80013889)$ & 217 & $15-09-63$ \\
\hline 23047010 & La Dorada & Limnimétrica & La Dorada & $(5,45,74,66666667)$ & 168 & $15-01-63$ \\
\hline 26160120 & La Cristalina & Pluviométrica & Neira & $(5,19219444,75,43577778)$ & 2411 & $15-09-70$ \\
\hline 23050130 & La Esperanza & Pluviométrica & Marquetalia & $(5,31666667,75,05)$ & 1400 & $15-08-66$ \\
\hline 26160160 & La María & Pluviométrica & Aguadas & $(5,5875,75,5775)$ & 1480 & $15-01-62$ \\
\hline 23020080 & Manzanares & Pluviométrica & Manzanares & $(5,26561111,75,14408333)$ & 1974 & $15-01-70$ \\
\hline 23050080 & Marquetalia & Pluviométrica & Marquetalia & $(5,29891667,75,05747222)$ & 1550 & $15-02-63$ \\
\hline 26160100 & Neira & Pluviométrica & Neira & $(5,16316667,75,518444444)$ & 198 & $15-09-70$ \\
\hline 26160090 & Plaza Feria Pácora & Pluviométrica & Pácora & $(5,52244444,75,45508333)$ & 712 & $15-08-70$ \\
\hline
\end{tabular}




\begin{tabular}{|c|c|c|c|c|c|c|}
\hline Código & Nombre & Tipo & Municipio & Coordenadas & Altitud & $\begin{array}{l}\text { Fecha de } \\
\text { instalación }\end{array}$ \\
\hline 26150150 & Papayal & Pluviométrica & Villamaría & $(4,95166667,75,48972222)$ & 2304 & $15-09-70$ \\
\hline 26160080 & Salamina Conc. Barc. & Pluviométrica & Salamina & $(5,39544444,75,48930556)$ & 1890 & $15-08-70$ \\
\hline 26185040 & San Félix & Climática ordinaria & Salamina & $(5,37294444,75,37533333)$ & 2690 & $15-08-70$ \\
\hline
\end{tabular}

b. Temperatura

\begin{tabular}{ll|l|l|l|ll} 
Código & Nombre & Tipo & Municipio & Coordenadas & Altitud & $\begin{array}{l}\text { Fecha de } \\
\text { instalación }\end{array}$ \\
\hline 26155110 & Aeropuerto La Nubia & Sinóptica principal & Manizales & $(5,02977778,75,46991667)$ & 2104 & $15-06-68$ \\
\hline 26145020 & Bellavista & Climática principal & Anserma & $(5,275944444,75,80013889)$ & 217 & $15-09-63$ \\
\hline 26155150 & Las Brisas & Climática principal & Villamaría & $(4,93452778,75,35038889)$ & 4133 & $15-10-81$ \\
\hline 26185040 & San Félix & Climática ordinaria & Salamina & $(5,37294444,75,37533333)$ & 2690 & $15-08-70$ \\
\hline
\end{tabular}

Fuente: Mintic (2020) [27].

Los datos obtenidos del repositorio de datos del Ideam contienen información diaria de precipitación, temperatura mínima y temperatura máxima, aun con valores faltantes que no fueron completados. Esta información fue transformada a mensual, mediante suma - en el caso de la precipitación - y calculando el promedio - en el caso de temperatura-. Se eligieron conjuntos de datos mensuales porque incluyen el análisis de variaciones mensuales a corto plazo, como los ciclos intra e interanuales [25]. Posteriormente, una revisión de la literatura permitió determinar el procedimiento para el análisis de tendencia en las series disponibles, el cual puede ser descrito genéricamente de la siguiente forma [28]:

- Se seleccionó la wavelet madre, longitud y niveles de descomposición.

- Se realizó la descomposición.

- Se graficaron la serie original, los detalles y la última aproximación.

- Se realizó un análisis gráfico de la tendencia.

En cuanto a la selección de la wavelet madre que se utilizaría, la revisión de antecedentes muestra que la wavelet Daubechies es ampliamente usada en estudios climáticos. De modo que fue seleccionada para el presente trabajo [25]. De igual forma, las wavelets suaves (de longitud cinco o superior) se prefieren, porque las tendencias se suponen graduales y representan procesos de cambio lento. Por esta razón, se seleccionó la wavelet Daubechies 5 (dB5). En cuanto a los niveles de descomposición, se usó la ecuación 5.

$$
L=\frac{\log \left(\frac{N}{2 v-1}\right)}{\log (2)}
$$

Donde:

$N$ es el número de datos de la serie, para este caso, 480 .

$v$ es la longitud de la wavelet madre. Ello da como resultado 5,773, es decir, seis niveles de descomposición [25].

Para el cálculo de la MRA se utilizó el software Matlab ${ }^{\oplus}$, específicamente función multi-level 1-D wavelet decomposition. Como se explicó en el referente teórico, la longitud de la serie para el análisis wavelet debe ser una potencia de dos. Dado que la potencia de dos más cercana es 512 , diversos métodos pueden ser utilizados para dicha extensión, tales como zero-padding, smooth padding, periodic extension, and boundary value replication (symmetrization) [29]. Esta adición puede generar una distorsión en la serie, conocida como efecto borde. De acuerdo con la revisión bibliográfica, el método 
de symmetrization produce el menor error relativo medio, al momento de reconstruir la señal. Por tal razón, fue el utilizado en este estudio [21].

\section{Resultados}

Para el análisis, se empleó como método la DWT, específicamente la wavelet Daubechies de longitud cinco (dB5) y nivel seis. Dado que se analizaron quince estaciones, solo se presenta como evidencia en esta sección la descomposición de la información de la estación Aeropuerto La Nubia para ambas variables; resultados similares se encontraron en las demás estaciones evaluadas.

En cuanto a los datos de precipitación, la figura 2 presenta la serie original, los detalles D1 a D6, así como la aproximación A6. La figura muestra que el componente de tendencia está contenido dentro de la parte de aproximación de la transformada wavelet, lo que implica que las tendencias están cambiando paulatina y gradualmente; y probablemente son de largo plazo. Las fluctuaciones bruscas pueden considerarse ruido y se reflejan en los detalles inferiores (D1-D3), mientras los detalles superiores pueden indicar periodicidades que afectan la tendencia.

En lo que se refiere a los datos de temperatura (discriminados en temperatura mínima y máxima), la figura 3 presenta la serie original, los detalles D1 a D6 y la aproximación A6. El análisis preliminar lleva a una conclusión similar a la precedente, la tendencia puede observarse en el componente de aproximación y, los detalles, que muestran las fluctuaciones bruscas, pueden considerarse ruido y se reflejan en los detalles inferiores (D1-D3), mientras que los detalles superiores pueden indicar periodicidades que afectan la tendencia.
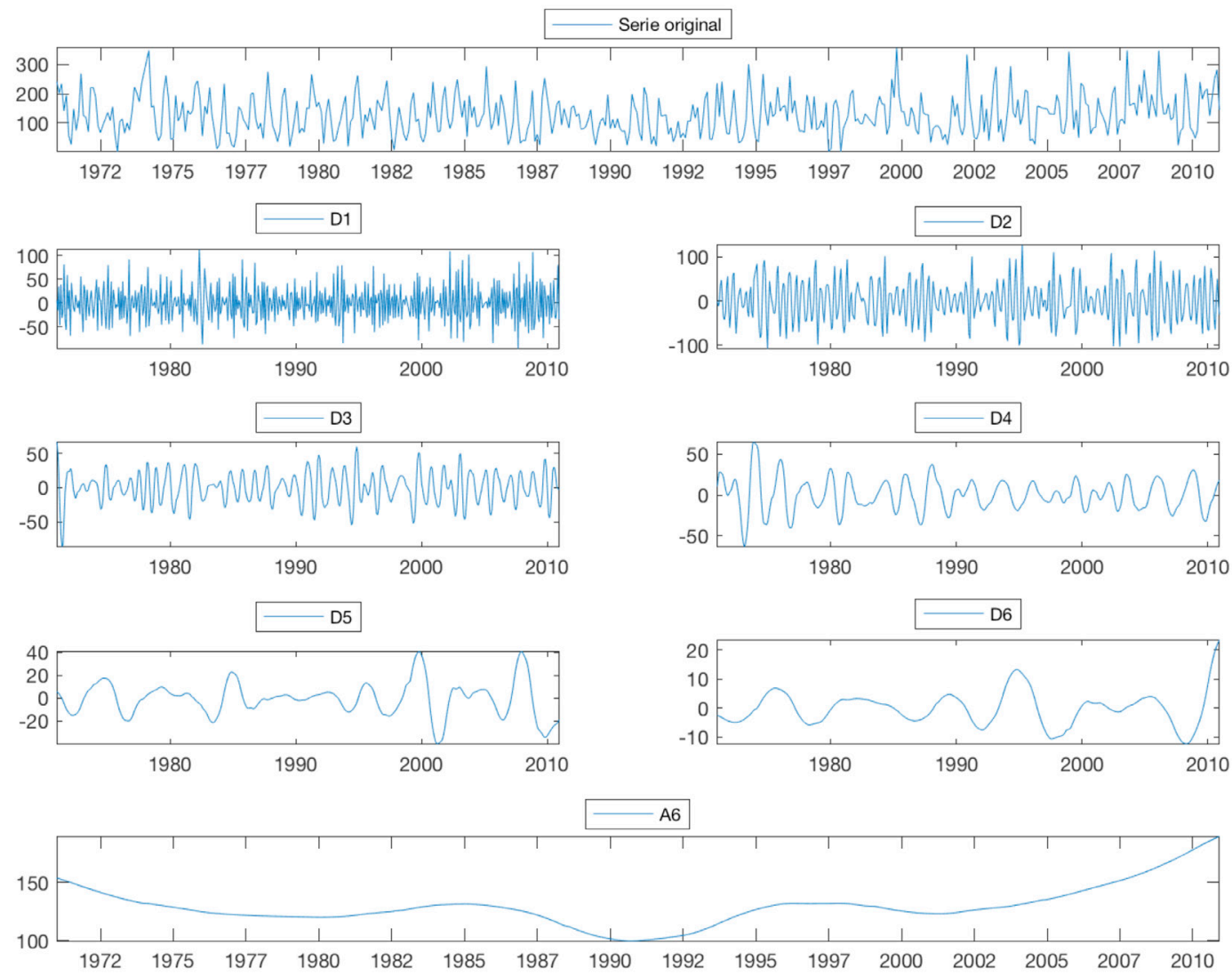

Figura 2. Serie de precipitación para la estación Aeropuerto La Nubia y su DWT, usando dB5 con seis niveles de descomposición (D1-D6) y una de aproximación (A6).

Fuente: elaboración propia. 


\section{a. Mínima}
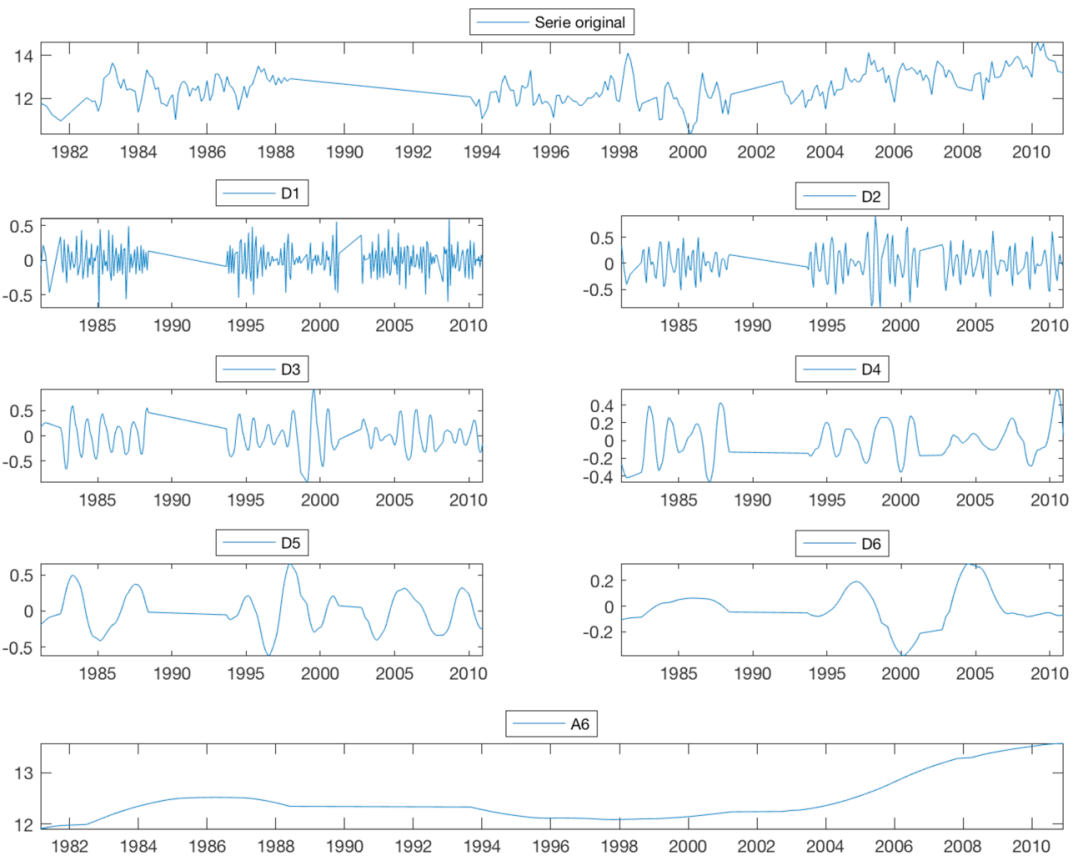

b. Máxima
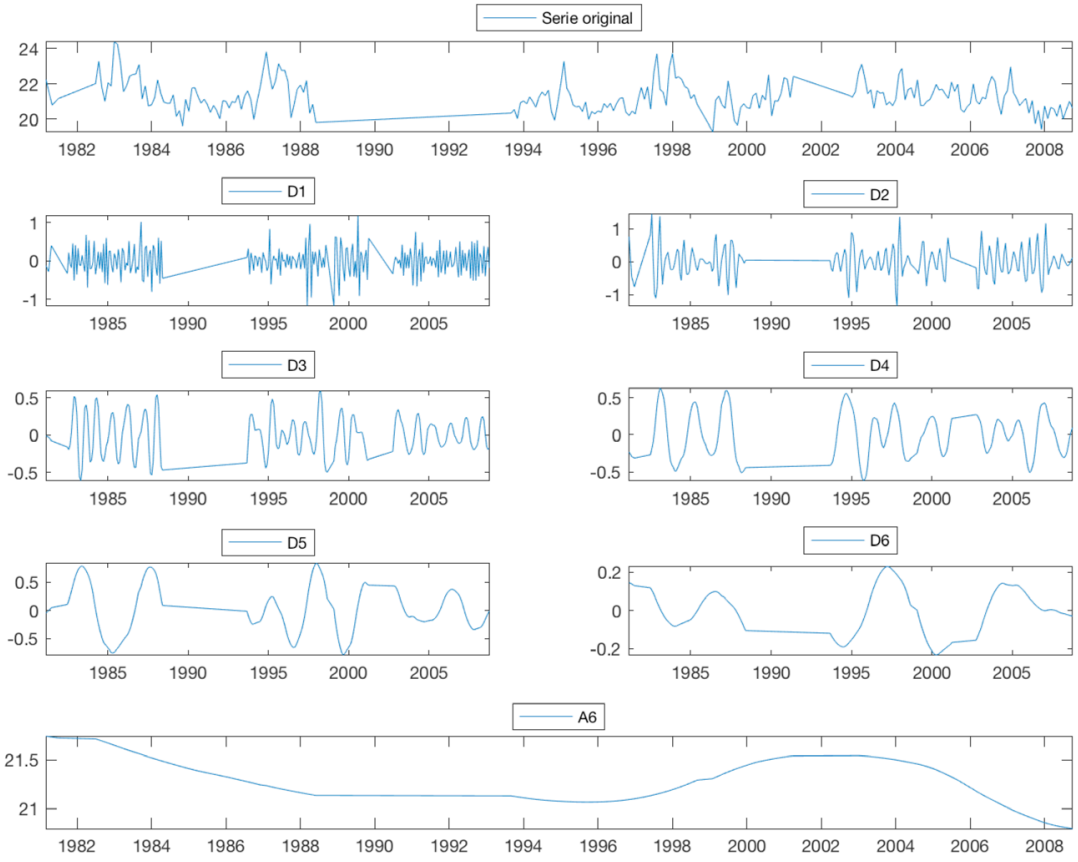

Figura 3. Series de temperatura (a) mínima y (b) máxima, para la estación Aeropuerto La Nubia y su DWT, usando dB5 con seis niveles de descomposición (D1-D6) y una de aproximación (A6).

Fuente: elaboración propia. 
La figura 4 consigna los resultados obtenidos en precipitación para las 15 estaciones analizadas, que muestran una tendencia creciente para todas las estaciones. La prueba clásica de Mann-Kendall detectó tendencias crecientes y significativas en las siguientes estaciones: Aeropuerto La Nubia, Aguadas, Bellavista, La Dorada, Manzanares, Marquetalia y Neira.

La figura 5 sintetiza el producto del análisis de la temperatura para las cuatro estaciones que hicieron parte de este estudio. Revela una tendencia lenta creciente en la temperatura mínima en todas las estaciones. Entretanto, para la temperatura máxima, se detectaron tendencias lentas decrecientes en el Aeropuerto La Nubia, aunque también se detectaron tendencias lentas crecientes en las demás estaciones.

El análisis de tendencias con la técnica no paramétrica clásica de Mann-Kendall permite confirmar tendencias crecientes en las temperaturas máximas en las siguientes estaciones: Bellavista, Las Brisas y San Félix. Al mismo tiempo, con esta prueba no paramétrica se confirmaron, además, tendencias positivas en las temperaturas mínimas para las mismas estaciones.
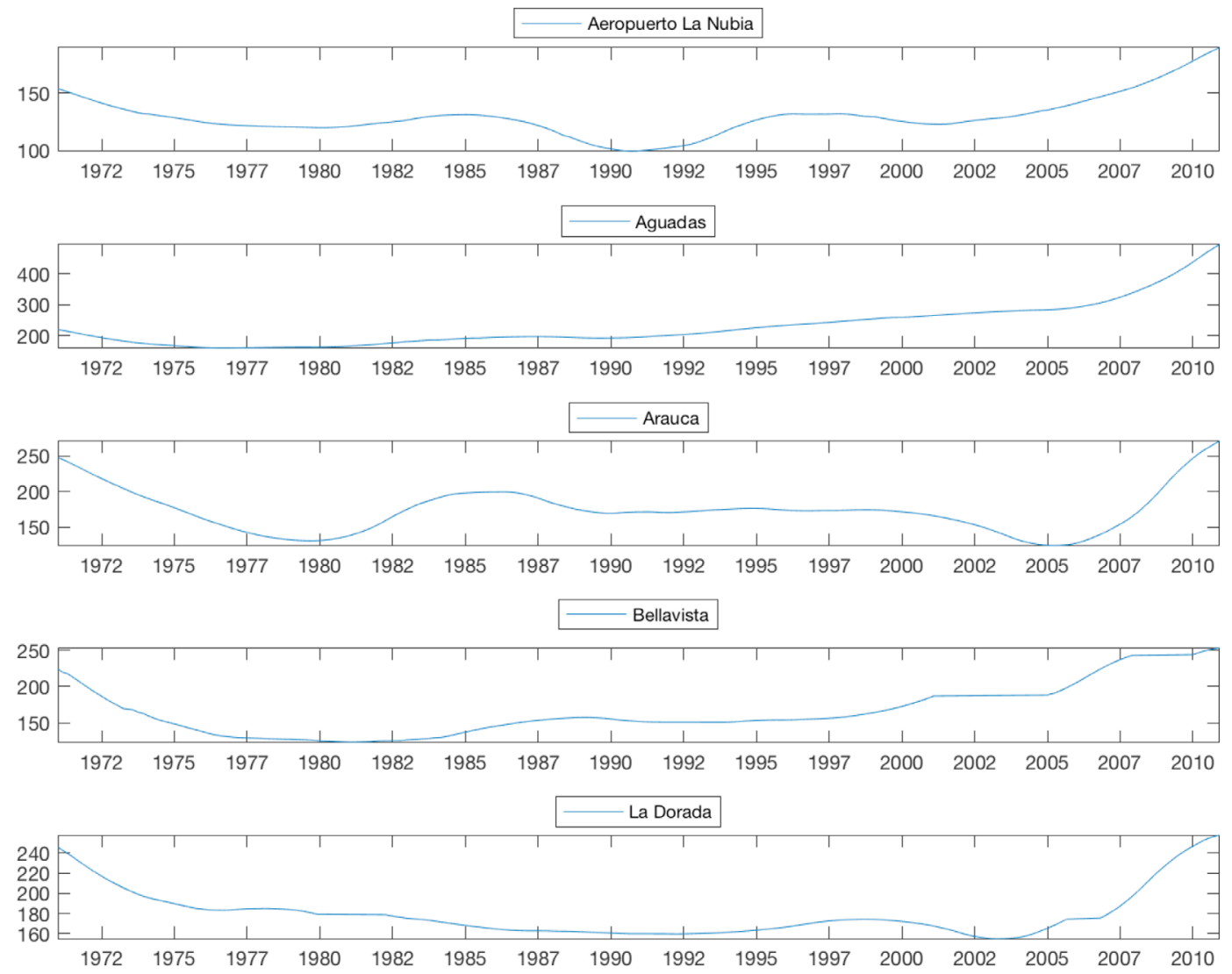

Figura 4. Tendencias en precipitación para las 15 estaciones analizadas.

Fuente: elaboración propia. 

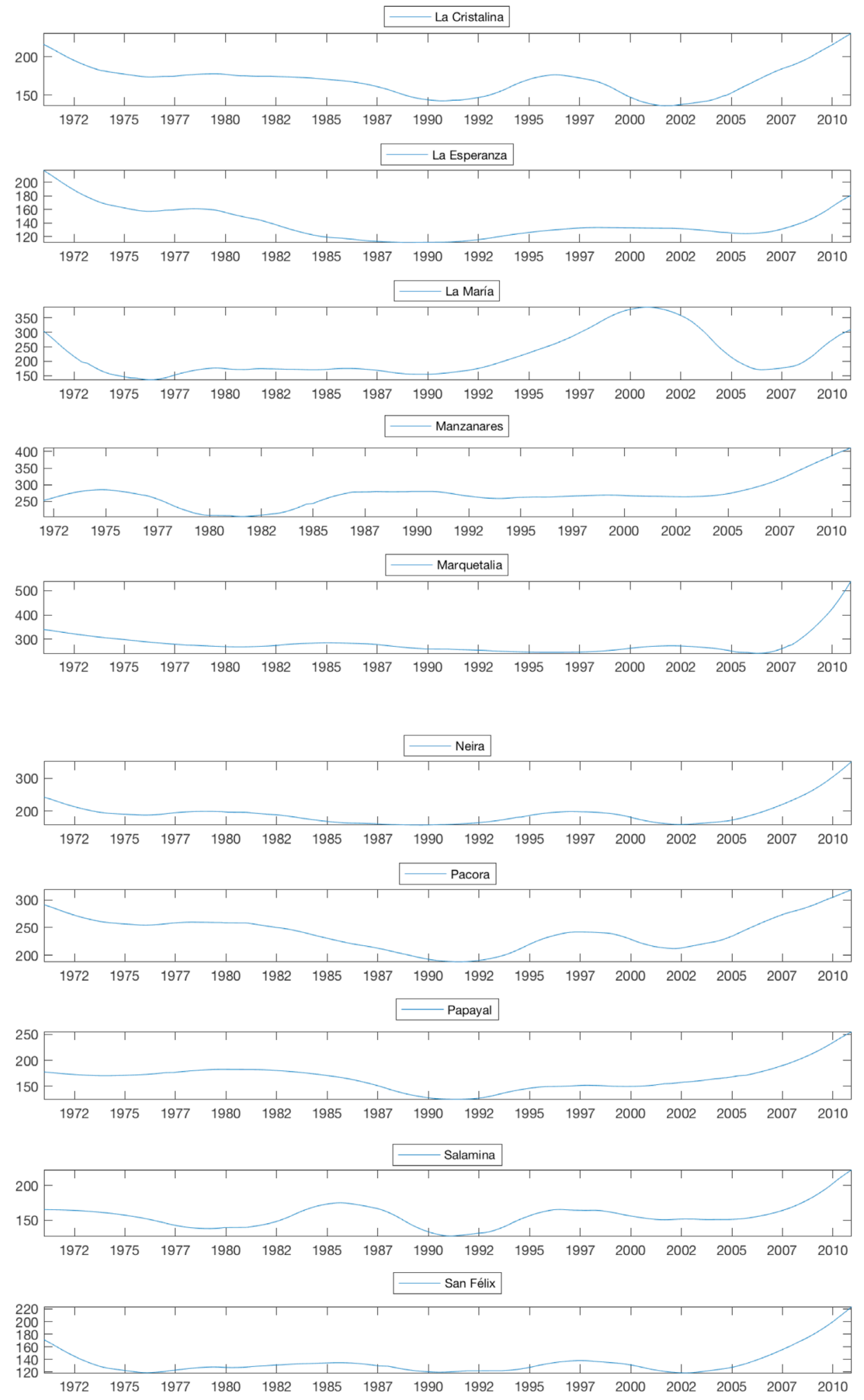

Figura 4. Tendencias en precipitación para las 15 estaciones analizadas (continuación).

Fuente: elaboración propia. 

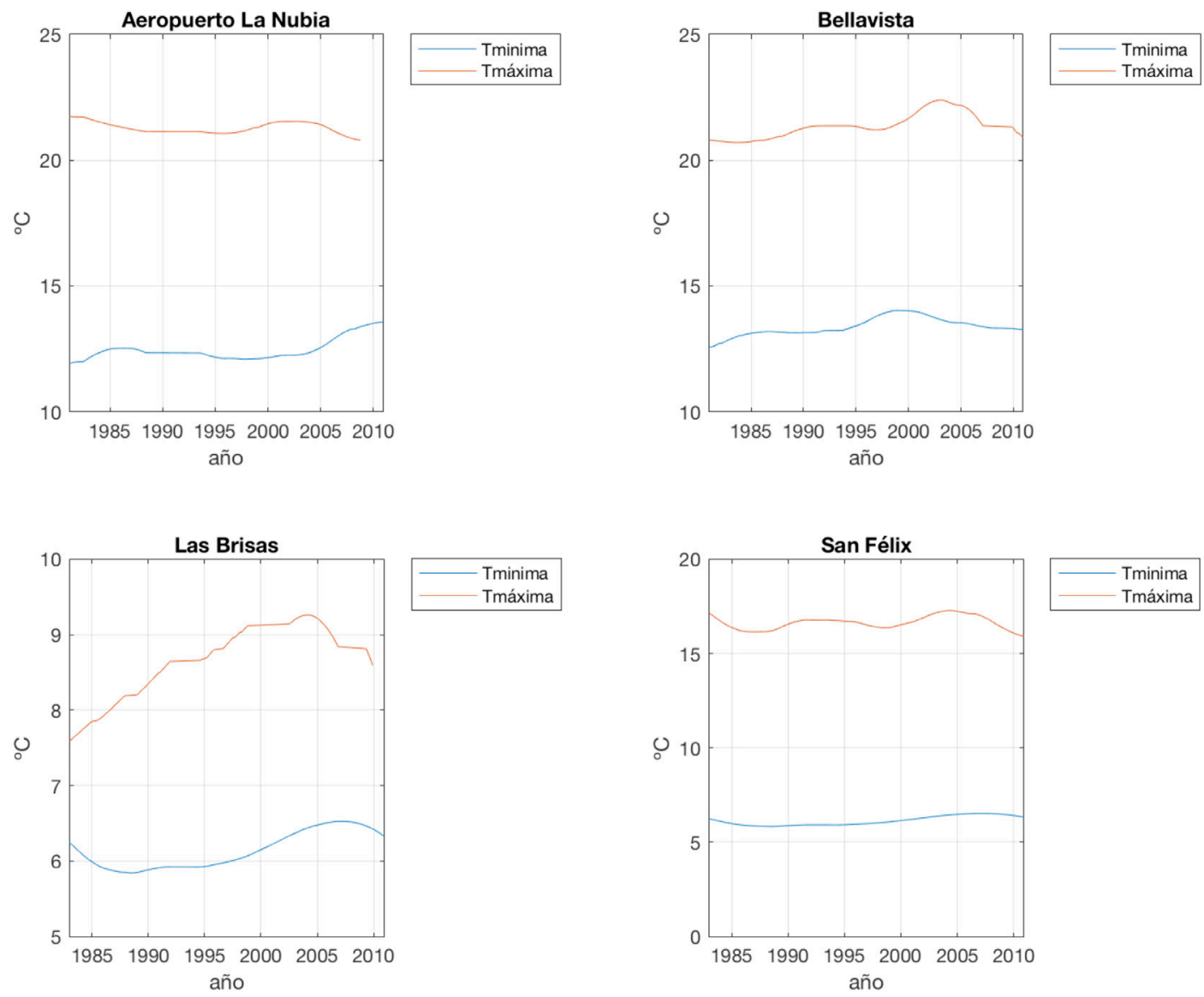

Figura 5. Tendencias en temperatura para las cuatro estaciones analizadas.

Fuente: elaboración propia.

En Colombia, diferentes estudios han detectado tendencias crecientes de largo plazo en la variable temperatura [30] y en las series de temperatura mínima [31] y máxima [32]. Para la región cafetera colombiana, Pérez y su equipo [33] identificaron tendencias crecientes en las series anuales de temperatura del aire con el estadístico de Mann-Kendall. Para el departamento de Caldas, el análisis de tendencias climáticas con RClimdex, realizado por Ocampo-López y su equipo [34], reportó tendencias crecientes solo en dos estaciones de diez analizadas para el valor mensual máximo de temperatura máxima diaria. Entretanto, para el valor mensual máximo de temperatura mínima diaria, se identificaron tendencias crecientes en seis estaciones de las diez evaluadas.
Por otra parte, los estudios para Colombia han reportado un aumento generalizado en las precipitaciones para el periodo 1971-2010 con la herramienta RClimdex [35]. Para Caldas, el análisis con RClimdex identificó para la precipitación anual total y el número de días húmedos, tendencias crecientes en 22 estaciones de 55 evaluadas [34]; se confirman tendencias crecientes en Aguadas y San Félix que fueron estaciones también evaluadas en este estudio. No obstante, los resultados de tendencias reportados aquí coinciden con estudios previos para esta región colombiana [36].

\section{Conclusión}

Pueden utilizarse múltiples técnicas para el análisis de tendencia de series de datos no estacionarias. 
Herramientas estadísticas como la prueba no paramétrica de Mann-Kendall son ampliamente utilizadas, así como el cálculo del periodograma, usando el análisis de Fourier, tanto la transformada de Fourier (FT) como la transformada de Fourier de tiempo reducido (STFT, por sus siglas en inglés). Este último método ofrece mejores resultados que la FT. Sin embargo, se queda corto en el análisis al tener una ventana de longitud fija [25].

Como herramienta, el análisis de Fourier no permite discernir comportamientos cíclicos de corta duración en la series analizadas. Este análisis puede resultar útil para series de datos diarios o estacionales donde pueden emerger este tipo de comportamientos periódicos. La descomposición wavelet (MRA), en cambio, arroja una tendencia lenta y gradual en ambas variables, precipitación y temperatura, a la luz de las estimaciones por la sexta aproximación. Las descomposiciones (detalles) de nivel más bajo evidencian el ruido presente en las series de datos y como es eliminado, si bien las de orden superior pueden evidenciar comportamientos cíclicos de corta duración.

Los resultados obtenidos en este trabajo son consistentes con la revisión de antecedentes [34][37], así como con lo plasmado en las Agendas $\mathrm{Mu}-$ nicipales y Departamental de Cambio Climático para Caldas, en cuanto a las líneas base de precipitación y temperatura [38].

El análisis sobre la variable precipitación arrojó una tendencia lenta y positiva para las estaciones estudiadas. En cuanto a temperatura, la tendencia fue lenta y positiva para la temperatura mínima; mientras que para la temperatura máxima las tendencias crecientes no fueron evidentes en todas las estaciones evaluadas. El uso de una wavelet más suave (como en el caso de la dB5 usada aquí) permite identificar mejor tendencias en datos climáticos, ya que se supone que las variaciones son graduales y de cambios lentos.

Finalmente, como trabajo futuro, para el análisis de tendencia es recomendable incorporar herramientas tradicionales al análisis wavelet (análisis de autocorrelación y pruebas como Mann-Kendall), a fin de tener criterios adicionales para la selección de los niveles de la wavelet a usar para mejorar el filtrado. De igual forma, posibles correlaciones entre variables adicionales, no necesariamente climáticas (como el uso del suelo), podrían enriquecer los hallazgos de tendencia y ampliar el espectro hacia estudios de naturaleza predictiva.

\section{Referencias}

[1] Programa de las Naciones Unidas para el Desarrollo -PNUD, Informe sobre Desarrollo Humano 2019. Más allá del ingreso, más allá de los promedios, más allá del presente. Desigualdades del desarrollo humano en el siglo XXI. Nueva York: Oficina del Informe sobre Desarrollo Humano del PNUD, 2020. Disponible en http:// hdr.undp.org/sites/default/files/hdr_2019_es.pdf

[2] Consejo Nacional de Política Económica y Social (Conpes), "Política de Crecimiento Verde (Conpes 3934)", 2018. Disponible en https://colaboracion.dnp. gov.co/CDT/Conpes/Econ\%C3\%B3micos/3934.pdf

[3] O. Ocampo, "El cambio climático y su impacto en el agro", Revista De Ingeniería, n. 33, pp. 115-123, 2011. Doi: https://www.doi.org/10.16924/riua.v0i33.184

[4] Redacción Medioambiente, "Así frenó la economía el fenómeno de El Niño”, El Espectador, 2018 [En línea]. Disponible en: https://www.elespectador.com/ noticias/medio-ambiente/asi-freno-la-economia-el-fenomeno-de-el-nino-articulo-803891 [Accedido: 06Mar-2020].

[5] Colprensa, "Por fenómeno de El Niño, ganaderos piden prórrogas para pago de créditos”, La Patria, 2014 [En línea]. Disponible en: http://www.lapatria.com/nacional/ por-fenomeno-de-el-nino-ganaderos-piden-prorrogas-para-pago-de-creditos-109841 [Accedido: 06-Mar2020].

[6] S. Turbay et al., "Adaptación a la variabilidad climática entre los caficultores de las cuencas de los ríos Porce y Chinchiná, Colombia”, Investigaciones Geográficas, no. 85, pp. 95-112, 2015. Doi: https://www.doi. org/10.14350/rig.42298

[7] G. Poveda et al., "Socio-environmental Vulnerability and Adaptation to Climate Change in the Coffee Growing Communities of the Central Andes of Colombia”, En Vulnerability Studies in the Americas: Extreme Weather and Climate Change, P. Musseta and M. Hurlbert, Eds. Cambridge (MA): Cambridge Scholars Publishing, 2020, pp. 158-180.

[8] P. Mussetta et al., "Vulnerabilidad al cambio climático. Dificultades en el uso de indicadores en dos cuencas de Colombia y Argentina”, Empiria. Revista de Metodología de Ciencias Sociales, no. 36, p. 119-147, 2017. Doi: https://www.doi.org/10.5944/empiria.36.2017.17862 
[9] Ideam, PNUD, DNPC-MADs, "Resumen ejecutivo Tercera Comunicación Nacional de Colombia a la Convención Marco de las Naciones Unidas sobre Cambio Climático (cmnucc)". Tercera Comunicación Nacional de Cambio Climático. Bogotá, Colombia. 2017.

[10] Intergovernmental Panel on Climate Change -IPCC. Summary for Policymakers. En: Global Warming of $1.5{ }^{\circ} \mathrm{C}$. An IPCC Special Report on the Impacts of Global Warming of $1.5^{\circ} \mathrm{C}$ above Pre-Industrial Levels and Related Global Greenhouse Gas Emission Pathways, in the Context of Strengthening the Global Response to the Threat of Climate Change, Sustainable Development, and Efforts to Eradicate Poverty. 2018. [V. Masson, P. Zhai, H. O. Pörtner, et al. (Eds.)]. World Meteorological Organization, Ginegra, Suiza, 32 pp. Disponible en https:/www.ipcc.ch/site/assets/uploads/sites/ 2/2019/05/SR15_SPM_version_report_LR.pdf

[11] J. L. Useros, "El cambio climático: sus causas y efectos medioambientales", Anales de la Real Academia de Medicina y Cirugía de Valladolid, vol. 50, pp. 71-98, 2012.

[12] L. Alexander, et al. "Global Observed Changes in Daily Climate Extremes of Temperature and Precipitation", Journal of Geophysical Research, vol. 111, n. ${ }^{\circ}$, 2006. Doi: https://www.doi.org/10.1029/2005jd006290

[13] M. Collins et al. "Long-term Climate Change: Projections, Commitments, and Irreversibility". En Climate Change 2013: The Physical Science Basis. Contribution of Working Group I to the Fifth Assessment Report of the Intergovernmental Panel on Climate Change, T. F. Stocker et al. Eds. Cambridge (MA): Cambridge University Press, 2013, pp. 1029-1136. Doi: https://www. doi.org/10.1017/CBO9781107415324.024

[14] Instituto de Hidrología, Meteorología y Estudios Ambientales -Ideam, "Escenarios de cambio climático Ideam”, Ideam.gov.co, 2020 [En línea]. Disponible en: http://www.ideam.gov.co/web/tiempo-y-clima/escenarios-cambio-climatico [Accedido: 06-May-2020].

[15] K. Trenberth, "Changes in Precipitation with Climate Change”, Climate Research, vol. 47, no. 1, pp. 123-138, 2011. Doi: https://www.doi.org/10.3354/cr00953

[16] H. Shiogama et al., "Emission Scenario Dependency of Precipitation on Global Warming in the MIROC3.2 Model", Journal of Climate, vol. 23, no. 9, pp. 2404-2417, 2010. Doi: https://www.doi.org/10.1175/2009jcli3428.1

[17] J. Corredor, "Análisis preliminar de precipitación máxima en Colombia”, Ciencia e Ingeniería Neogranadina, vol. 17, no. 1, pp. 79-93, 2007. Doi: https://www. doi.org/10.18359/rcin.1087
[18] J. Corredor y V. Peñaranda, "Identificación de los parámetros del modelo del número de curva y su incertidumbre mensual en la cuenca alta del río Bogotá", Ciencia e Ingeniería Neogranadina, vol. 22, no. 1, pp. 75, 2012. Doi: https://www.doi.org/10.18359/rcin.250

[19] O. Castellanos-Domínguez, A. Fúquene-Montañez y D. Ramírez-Martínez, Análisis de tendencias: de la información hacia la innovación. Bogotá: Universidad Nacional de Colombia, 2011.

[20] E. Moreno-Sarmiento, "Predicción con series de tiempo y regresión”, Panorama, vol. 2, no. 4, 2013. Doi: https://www.doi.org/10.15765/pnrm.v2i4.262

[21] L. Castro y Y. Escobar, "Análisis de tendencia y homogeneidad de series climatológicas”, Ingeniería de Recursos Naturales y del Ambiente, 2010, n. ${ }^{\circ}$ 9, pp. 15-25.

[22] A. Velasco-Rivera y M. Granados-Ortiz, "Tendencias e incidencia de los fenómenos macroclimáticos en la hidrología del alto y medio Magdalena”, Ciencia e Ingeniería Neogranadina, vol. 16, n. ${ }^{\circ}$ 2, pp. 29-44, 2006. doi: https://www.doi.org/10.18359/rcin.1231

[23] A. Araghi, M. Mousavi Baygi, J. Adamowski, J. Malard, D. Nalley and S. Hasheminia, "Using wavelet transforms to estimate surface temperature trends and dominant periodicities in Iran based on gridded reanalysis data”, Atmospheric Research, vol. 155, pp. 52-72, 2015. doi: https://www.doi.org/10.1016/j.atmosres.2014.11.016

[24] C. Torrence y G. Compo, "A Practical Guide to Wavelet Analysis", Bulletin of the American Meteorological Society, vol. 79, n. ${ }^{\circ}$ 1, pp. 61-78, 1998. Doi: https:// www.doi.org/10.1175/1520-0477(1998)079<0061:apgtwa>2.0.co;2

[25] D. Nalley, J. Adamowski y B. Khalil, "Using discrete wavelet transforms to analyze trends in streamflow and precipitation in Quebec and Ontario (1954-2008)", Journal of Hydrology, vol. 475, pp. 204-228, 2012. Doi: https://www.doi.org/10.1016/j.jhydrol.2012.09.049

[26] N. Olivera, Wavelets de Haar y Daubechies y sus aplicaciones, Tesis de grado, Matemática Computacional, Universidad Jaume I, Castellón de la Plana, España, 2018. Disponible en http://hdl.handle. net/10234/177642

[27] Ministerio de Tecnologías de la Información y Comunicaciones -MinTIC, "Datos Abiertos - Catalogo Estaciones Ideam”, Datos.gov.co, 2020 [En línea]. Disponible en: https://www.datos.gov.co/Ambiente-y-Desarrollo-Sostenible/Catalogo-Estaciones-IDEAM/ n6vw-vkfe. [Accedido: 06-Mar-2020]. 
[28] A. Rahman, A. Anik, Z. Farhana, S. Devnath y Z. Ahmed, "Pattern Recognition of Rainfall Using Wavelet Transform in Bangladesh", Open Journal of Statistics, vol. 08, n. ${ }^{\circ}$ 01, pp. 134-145, 2018. Doi: https://www.doi. org/10.4236/ojs.2018.81009

[29] Mathworks, "Border Effects- Matlab \& SimulinkMathWorks América Latina", La.mathworks.com, 2020 [En línea]. Disponible en: https://la.mathworks. com/help/wavelet/ug/dealing-with-border-distortion. html [Accedido: 06-Mar-2020].

[30] J. D. Pabón, "Cambio climático en Colombia: tendencias en la segunda mitad del siglo XX y escenarios posibles para el siglo XXI", Rev. Acad. Colomb. Cienc., vol. 36, n. ${ }^{\circ} 139$, pp. 262-278, 2012.

[31] G. Hurtado, Análisis del comportamiento promedio y tendencias de largo plazo de las temperaturas mínimas medias para las regiones hidroclimáticas de Colombia. Bogotá: Ideam, 2012.

[32] G. Hurtado, Análisis del comportamiento promedio y tendencias de largo plazo de la temperatura máxima media para las regiones hidroclimáticas de Colombia, Bogotá: Ideam, 2012.

[33] E. P. Pérez, V. H. Ramírez y A. J. Peña, "Variabilidad espacial y temporal de la temperatura del aire en la zona cafetera colombiana”, Investigaciones Geográficas, Boletín del Instituto de Geografía, vol. 89, n. . 2016, pp. 23-40, 2016. Doi: https://www.doi.org/10.14350/ rig. 38707
[34] O. L. Ocampo-López, J. J. Vélez-Upegui, T. Forero-Hernández y J.P. Marín-Salazar, "Análisis de tendencias climáticas on RClimdex en el departamento de Caldas Colombia”, Sci. T., vol. 25, n. ${ }^{\circ}$ 4, pp. 595-603, 2020.

[35] R. Mayorga, G. Hurtado y H. Benavides, "Evidencias de cambio climático en Colombia con base en información estadística”, Nota Técnica Ideam, Bogotá, 2011.

[36] O. Ocampo, Modelación hidrológica y agronómica de los efectos del cambio y la variabilidad climática en la producción cafetera de Caldas, Tesis doctoral, Ingeniería Línea Automática, Universidad Nacional de Colombia, Manizales Colombia, 2018. Disponible en https://repositorio.unal.edu.co/handle/unal/63694 [Accedido: 05-May-2021].

[37] E. Hernández-Leal, N. Duque-Méndez y J. MorenoCadavid, "Generación de pronósticos para la precipitación diaria en una serie de tiempo de datos meteorológicos”, Ingenio Magno, vol. 7, n. ${ }^{\circ}$ 1, pp. 144-155, 2006 http://revistas.ustatunja.edu.co/index.php/ingeniomagno/article/view/1172

[38] Corporación Autónoma de Caldas -Corpocaldas, “Corpocaldas -Cambio Climático -Agendas Climáticas Municipales”, Corpocaldas.gov.co, 2019 [En línea]. Disponible en: http://www.corpocaldas.gov.co/dynamic_page.aspx?p=1598. [Accedido: 10-Jun-2020]. 